\title{
eJRIEPS
}

Ejournal de la recherche sur l'intervention en éducation physique et sport

$18 \mid 2009$

Varia

\section{Apprentissage et développement dans la perspective de l'interactionnisme socio-discursif}

Jean-Paul Bronckart

\section{(2) OpenEdition}

\section{Journals}

Édition électronique

URL : http://journals.openedition.org/ejrieps/5476

DOI : 10.4000/ejrieps.5476

ISSN : 2105-0821

Éditeur

ELLIADD

\section{Référence électronique}

Jean-Paul Bronckart, «Apprentissage et développement dans la perspective de l'interactionnisme socio-discursif », eJRIEPS [En ligne], 18 | 2009, mis en ligne le 01 juillet 2009, consulté le 18 mars 2021. URL : http://journals.openedition.org/ejrieps/5476 ; DOI : https://doi.org/10.4000/ejrieps.5476

\section{(c) (1)}

La revue eJRIEPS est mise à disposition selon les termes de la Creative Commons Attribution 4.0 International License. 
Apprentissage et développement dans la perspective de l'interactionnisme sociodiscursif

Jean-Paul Bronckart

FPSE, Sciences de l'Education. Université de Genève

Ce texte est la conférence prononcée par Jean-Paul Bronckart au Séminaire International de l'AIESEP à Besançon le 29 mai 2009.

Comme le montrent les documents de présentation, ce séminaire international met l'accent sur trois thèmes et/ou thèses d'une approche constructiviste de la pédagogie du sport : - le caractère actif de l'élaboration des connaissances ; - le caractère situé des actions aussi bien que des apprentissages ; - l'importance de la réflexion sur l'action dans la formation en EPS (comme en d'autres formations de professionnels).

Notre position propre concernant ces thèses peut se résumer de la manière suivante.

- La réflexion sur l'action constitue indiscutablement un important moyen de construction des connaissances, mais ce n'est néanmoins pas l'unique instrument de cette construction, et par ailleurs sa mise en œuvre requiert une sérieuse maîtrise théorique et méthodologique.

- Les trois concepts centraux de l'approche proposée méritent réflexion et discussion : quelle est la signification précise des notions d'action (ou d'agir, ou d'activité), de réflexion et de caractère "situé" (des actions et des apprentissages) ?

- Le langage est l'instrument majeur de la réflexion. Son rôle n'est cependant pas de refléter (comme en miroir) les entités représentées, mais de contribuer à l'élaboration même ou au façonnage de ces entités, et il convient donc d'analyser soigneusement ces effets structurants du langage sur les processus et les produits de la réflexion.

Notre intervention visera à développer quelques aspects de cette position, selon le schéma qui suit. Nous expliciterons d'abord les principes généraux de notre cadre théorique, à savoir l'interactionnisme socio-discursif, qui est une variante de l'interactionnisme social. Nous examinerons ensuite les diverses conceptions théoriques de l'action, en tant qu'objet de la réflexion, et sur cette base nous procéderons à une analyse des dispositifs d'analyse des pratiques actuellement utilisés en formation des adultes. Nous évoquerons alors nos travaux ayant trait au langage et au rôle qu'il joue dans les processus réflexifs, et nous terminerons en posant le problème des conditions sous lesquelles la réflexion ou la prise de conscience peut être source d'apprentissage et de développement. 


\section{L'interactionnisme socio-discursif (ISD)}

La démarche de I'ISD se situe dans le prolongement du mouvement interactionniste social qui avait émergé au premier tiers du XXe, dans le cadre des œuvres de Bühler (1927), Dewey (1925), Mead (1934), Voloshinov (1929/1977), Vygotski (1934/1997) et bien d'autres.

Par opposition aux autres paradigmes psychologiques (behaviorisme, constructivisme, cognitivisme), l'interactionnisme social met un accent prioritaire sur la sociohistoire humaine et sur les formes d'activités collectives élaborées par les groupes au cours de cette même histoire, parce qu'il considère que ces activités constituent les cadres médiateurs de l'ensemble des interactions entre les humains et leur milieu. II prend aussi résolument en compte le rôle que jouent les activités éducatives et/ou formatives dans le développement et, à la suite de Leontiev (1979), soutient que l'ensemble des connaissances humaines sont les produits de la réfraction psychique de ces interactions et de leurs objets. L'interactionnisme socio-discursif (Bronckart, 1997 ; 2008) se centre plus particulièrement sur les conditions effectives de fonctionnement et d'organisation du langage, et analyse le rôle que jouent les pratiques langagières dans la constitution et le développement de la pensée ; en outre, à la suite de Durkheim (1898) et de Saussure (2002), il pose que les constructions psychiques humaines ont deux lieux d'ancrage en perpétuelle interaction : elles s'organisent d'une part en représentations collectives soutenues par la langue externe ou commune, d'autre part en représentations individuelles soutenues par la langue interne de chaque personne.

Le programme de travail de I'ISD porte dès lors sur trois domaines, abordés dans une perspective "descendante".

Le premier domaine est celui des préconstruits issus de l'histoire sociale, au sein desquels il convient de distinguer les préconstruits praxéologiques (de l'ordre du "faire") et les préconstruits gnoséologiques (de l'ordre des connaissances et/ou savoirs).

Au plan praxéologique, il y a d'un côté les diverses formes d'activités pratiques collectives, telles qu'elles ont été élaborées et qu'elles sont gérées par les différentes formations sociales (les activités sportives qui nous concernent constituent un exemple de ce premier type de préconstruit praxéologique). II y a d'un autre côté les activités langagières, qui exploitent les ressources d'une langue naturelle et qui se réalisent empiriquement en textes, oraux ou écrits; activités dont la fonction première est, comme le soulignait Habermas (1987), de permettre l'entente dans la réalisation des activités pratiques (les textes servent à planifier, à réguler et à évaluer ces activités). Ces deux sortes d'activités se caractérisent surtout par leur diversité: diversité quasi infinie des activités pratiques, et diversité importante, mais plus limitée, des genres de textes en usage dans une communauté 
linguistique. Elles se caractérisent aussi par leur historicité (elles se transforment de générations en générations), leur socialité (elles sont construites, gérées, revendiquées par des formations sociales particulières, parfois dans des contextes de compétition ou de conflit) et leur culturalité (elles sont investies de valeurs, de normes et de règles propres à un groupe et contribuant à la confection de l'identité de ce même groupe).

Le plan gnoséologique est constitué des formes de connaissances élaborées au cours de l'histoire, ou encore des représentations collectives, qui, selon Popper et Habermas, sont de trois types ou relèvent de trois mondes formels : - le monde objectif, qui rassemble et organise les connaissances relatives à l'univers dans ses dimensions matérielles (connaissances évaluables sur la base des critères de vérité et d'efficacité) ; - le monde social, qui rassemble et organise les connaissances relatives aux modalités d'interactions entre les humains (connaissances évaluables sur la base du critère de la conformité aux normes) ; - le monde subjectif, qui rassemble et organise les connaissances relatives à la structure psychique des personnes (connaissances évaluables sur la base du critère de sincérité ou d'authenticité). Ces préconstruits tendent à l'universalité, parce que leur élaboration repose sur la mise en œuvre de processus cognitifs eux mêmes universaux, notamment de l'abstraction et de la généralisation au sens de Piaget (1970).

Le deuxième domaine est celui des démarches de médiation formative ou des entreprises d'éducation, informelle ou formelle, par lesquelles des éléments des préconstruits sont présentés aux apprenants, dans le double but de leur transmettre ces acquis et de leur permettre de contribuer ensuite à leur transformation ou à leur enrichissement. Une part de nos propres recherches est centrée sur les systèmes formels d'enseignement, notamment sur les textes d'orientation (parfois qualifiés de textes prescriptifs) qui y sont produits, et nous analysons les modalités de présentation de trois types d'objets de formation : - les objets épistémiques (résultant de la transposition didactique de certains aspects du monde objectif) ; - les objets praxéologiques (capacités d'action ou compétences en regard des attentes du monde social) ; - les objets psychologiques (qualités identitaires, éthiques, de caractère, etc., requises des personnes singulières, selon les conceptions en vigueur du monde subjectif).

Le troisième domaine concerne les effets produits par ces démarches formatives sur la constitution et le développement des personnes. L'ISD ne retient pas la notion de "sujet", en raison de sa connotation cartésienne de toute-puissance et d'universalité ; il adopte par contre résolument celle de personne, pour souligner la radicale singularité de la structure psychique de chaque individu, conséquence de l'inévitable singularité de son histoire de formation. La personne est abordée dans une perspective ne niant pas l'existence de 
capacités psychiques et comportementales innées, mais visant à mettre en évidence les transformations décisives de la teneur et de l'organisation de ces mêmes capacités qui sont provoquées par l'appropriation des préconstruits. Dans cette perspective, le développement est conçu comme le produit de la contradiction - ou du conflit - entre l'état actuel des connaissances ou pratiques d'une personne et les nouveautés qui, sur ces deux plans, sont proposées par l'entourage: l'enseignement prépare à la contradiction en créant et commentant des situations problèmes, mais c'est l'apprenant qui se développe, lorsqu'il peut intérioriser ces nouveautés et transformer ce faisant son organisation psychique.

\section{L'action et ses angles de saisie}

Quel est l'objet visé par l'analyse des pratiques, ou en d'autres termes, qu'est-ce que l'action?

En première approche, cette notion désigne une forme d'interprétation de l'activité, visant à y découper une séquence comportementale attribuable à une personne. Si elles sont multiples, les conceptions de l'action nous paraissent néanmoins pouvoir être regroupées en quatre rubriques.

La première est issue d'une longue tradition en philosophie, puis dans les sciences économiques, qui pose l'existence d'un acteur rationnel, ayant toutes les ressources nécessaires pour agir de manière efficace. Cette conception a été reprise par la philosophie analytique, en particulier par Anscombe (1957/2001), dont le propos était de distinguer l'action sensée des simples événements se produisant dans la nature. Mobilisant la technique de " déchiffrement logique des énoncés ", elle considère qu'un énoncé comme Pierre fait tomber deux tuiles du toit pour endommager la voiture du voisin qu'il déteste relate un enchaînement de phénomènes qui peut être saisi, soit comme événement, soit comme action. Dans le premier cas, on considèrera que l'occurrence du phénomène a (Pierre fait tomber les tuiles) est la condition nécessaire et suffisante pour celle du phénomène $b$ (la voiture du voisin est endommagée). Dans le second cas, on prendra en compte les propriétés psychiques imputées à Pierre, en l'occurrence l'évocation de l'existence d'un motif (Pierre déteste son voisin) et d'une intention (Pierre fait tomber ... pour), et on s'interrogera sur les relations existant entre ces propriétés psychiques de l'agent et celles des phénomènes observables. C'est à cette condition que l'enchaînement relaté peut être saisi en tant qu'action. La sémantique de l'action de Ricœur (1977) codifie cette approche en posant que toute action se définit comme une intervention d'un acteur dans le monde, acteur disposant de capacités comportementales et mentales, assumant des motifs ou raisons (le "pourquoi" du faire) ainsi que des intentions (les effets escomptés du faire), et étant ce faisant responsable de son 
action. Ce type d'approche saisit donc l'action sous l'angle exclusif des propriétés de l'acteur au moment de son intervention, sans prendre en compte, ni le déroulement de l'agir, ni les divers facteurs externes susceptibles de le réorienter, ni les résultats auxquels il aboutit.

Une deuxième approche met l'accent sur les déterminations externes et conteste que l'action humaine puisse s'appréhender en tenant compte des seules propriétés de l'agent/acteur. Pour Habermas (1987), tout agir humain se déploie en regard de représentations collectives qui sont organisées dans les trois mondes formels évoqués plus haut. En un état synchronique donné, ces mondes constituent des systèmes de coordonnées formelles vis-à-vis desquels tout agir humain exhibe des prétentions à la validité, et à partir desquels s'exercent en retour des évaluations et/ou des contrôles collectifs. Ces évaluations, qui s'effectuent par le biais du langage (de l'“agir communicationnel") sont une source d'autocompréhension, ou encore font passer certaines entités psychiques, du niveau du monde vécu à celui des représentations partageables.

Une troisième approche saisit l'action sous l'angle de son déroulement ou de sa dynamique. Schütz (1998) distingue notamment l'action accomplie de l'action en train de se faire, caractérisée par la temporalité vécue de l'actant. Selon cet auteur, chaque actant attribue à son agir un sens initial (issu d'un acte réfléchi), mais celui-ci se modifie au cours de l'agir, dans des directions a priori imprévisibles ; l'action se déploie en d'autres termes selon une temporalité interne dynamique, qui est à distinguer de la saisie temporelle rétrospective des observateurs, qui tend à conférer à cette même action unité et homogénéité. Bühler (1927) a pour sa part insisté aussi sur ce même cours temporel de l'action, conçu en termes de mécanismes de pilotage ou de régulation. Pour lui, les buts ou intentions imputables à l'actant ne coïncident en principe jamais avec le cours effectif de l'action, ou avec sa réalisation: l'actant se trouve en effet exposé à de multiples systèmes de connaissances ou de déterminations qui interfèrent avec le but qu'il s'est donné, et il se trouve de la sorte contraint de "piloter à vue". Dans cette conception, le cours de l'action est conçu comme un processus de négociation permanente entre les intentions initiales de l'actant et les déterminations multiples et contradictoires auxquelles il doit faire face.

Dans le cadre de sa théorie de la structuration, Giddens (1987) propose une quatrième approche, qui prend radicalement à contre-pied la précédente. Ce auteur souligne certes que l'actant exerce un contrôle réflexif sur l'activité en cours, qu'il procède en outre à une rationalisation de son agir et de celui des autres, et qu'il peut enfin se doter d'une motivation. Mais ces processus ne seraient pas pour autant définitoires de l'action ; pour Giddens, les motivations et les intentions dépendent de facteurs qui sont externes à l'agir même (la plupart de nos actions n'ont aucun motif particulier, et une action peut aboutir à des effets 
sans rapport avec l'intention initiale de l'actant), et en conséquence l'action serait exclusivement identifiable à son résultat: elle pourrait se définir comme une intervention humaine aboutissant effectivement à la modification d'un procès en cours.

\section{La formation par l'analyse des pratiques}

Dans le domaine de la formation des adultes, on peut repérer deux grands types d'ingénierie. Le premier rassemble les diverses démarches de transmission de savoirs préalablement répertoriés et formalisés. Le second, plus récent et au cœur du présent séminaire, rassemble les dispositifs d'analyse des pratiques, qui soumettent des exemples d'agir réel à des procédures de mise en intelligibilité. Quatre approches sont surtout exploitées en formation des adultes.

\section{a) Le praticien réflexif (Schön)}

Selon Schön, les professionnels font preuve d'un véritable « art de la pratique » qui ne peut pas s'enseigner, mais qui peut être appris (1983, p. 38). II s'agit d'un savoir contenu dans l'action, mais qui reste tacite, intuitif, non verbal, et par conséquent inaccessible à la description directe.

Schön distingue une « réflexion en cours d'action » qui peut émerger lorsque la situation confronte le praticien à l'indétermination, et une « réflexion sur l'action » qui est verbale, explicite, et consiste en un processus postérieur à l'action, à caractère généralement évaluatif.

La démarche de cet auteur consiste surtout en un plaidoyer pour que les formations «aident les professionnels à être plus réflexifs » au cours de leurs pratiques, mais ce plaidoyer n'est cependant pas assorti d'une clarification théorique de la nature même des processus réflexifs, et par ailleurs elle ne se concrétise en aucune méthode spécifique de formation.

\section{b) L'entretien d'explicitation (Vermersch)}

Cette méthode est fondée sur la conception de la subjectivité issue de la phénoménologie husserlienne et sur la conception piagétienne de la prise de conscience. Elle est définie comme une technique « non inductive d'aide à la prise de conscience » dont la spécificité est de « viser la verbalisation de l'action ».

L'objet de l'entretien d'explicitation est une action passée, effectivement réalisée, qui doit être re-saisie sous l'angle de son déroulement et telle qu'elle a été vécue, ressentie, par le sujet. Dans le cadre de cette démarche, diverses dimensions sont considérées comme des 
« satellites» ne relevant pas véritablement de l'action: les aspects conceptuels, généralisants, ou théoriques déclaratifs (savoirs, connaissances sur l'action en général, consignes, règles) ; les aspects émotionnels, affectifs et intentionnels; les résultats de l'action (cf. Vermersch, 1994).

L'intelligibilité visée consiste en un déplacement de l'objet-action du registre pré-réfléchi au registre réfléchi : transférée couche après couche, l'action est progressivement clarifiée.

\section{c) L'auto-confrontation (Clot \& Faïta)}

Développée dans le cadre de la clinique de l'activité de Clot (2001) et ancrée dans les théories de Leontiev et de Vygotski, cette technique consiste d'abord à filmer des séquences d'activité de travail, puis à présenter ces séquences aux travailleurs concernés et à organiser un débat réflexif au cours du visionnement du film de l'activité.

L'objet de l'auto-confrontation n'est pas le détail du déroulement vécu d'une action particulière, mais bien au contraire l'activité dans toute sa complexité, dans ses diverses modalités de conception et de réalisation par divers travailleurs, dans ses dimensions empêchées aussi bien qu'effectives.

L'intelligibilité visée est de l'ordre de la reconstruction de significations à propos de l'activité et plus généralement des situations de travail. L'auto-confrontation ne déclenche pas un rappel ou une re-évocation plus détaillée d'une action passée donnée (comme dans l'entretien d'explicitation), mais engendre un débat autour des conditions de réalisation du travail, qui fait émerger une nouvelle expérience de l'activité.

\section{d) L'instruction au sosie (Oddone $\rightarrow$ Clot)}

Inventée il y a trois décennies, cette technique visait à identifier « les règles de conduites » et « les stratégies individuelles » des travailleurs, de manière à accéder au « plan-programme » de chacun (Oddone, Re \& Briante, 1981, pp. 55-57). Elle a été reprise et transformée par Clot et ses collaborateurs, dans une perspective à la fois de formation et d'analyse du travail.

L'objet visé est l'activité de travail, à la fois dans sa globalité et dans ses menus détails ; elle porte en particulier sur les «ficelles du métier», les modes de comportements, les rapports aux collègues et à la hiérarchie, etc.

L'intelligibilité visée est de l'ordre de la reconstruction de significations à propos de l'activité et du métier, mais sous une modalité proactive ou planificatrice : c'est dans le cadre des instructions qu'il donne au sosie que le modèle prend conscience de propriétés effectives (ou possibles) de son activité de travail. 
Selon nous,

- les deux méthodes les plus utiles pour les apprentissages et le développement sont l'autoconfrontation et l'instruction au sosie ;

- l'adoption de ces démarches n'implique pas l'abandon des formes plus classiques de transmission de connaissances ;

- ces deux types de dispositifs sont en réalité complémentaires ; l'analyse des pratiques a notamment pour but de re-interroger les connaissances formelles, de leur faire "prendre vie" en les confrontant aux propriétés des situations effectives de travail.

\section{Une approche du langage}

\section{1. Le langage comme activité}

Le langage a une dimension primairement praxéologique, que Habermas (op. cit.) qualifiait d'« agir communicationnel », et que nous appelons « l'activité langagière ».

Cette activité exploite les ressources lexicales et syntaxiques propres à une langue naturelle, et se concrétise en textes, au sens contemporain du terme, c'est-à-dire en séquences organisées d'énoncés, écrits ou oraux.

Les textes ont des formes d'organisation diverses, notamment parce qu'ils s'articulent à des activités pratiques humaines elles-mêmes extrêmement diverses ; formes qui une fois stabilisées et décrites constituent les genres textuels.

Ces genres sont disponibles, à titre de modèles, dans cette rubrique des préconstruits collectifs que constitue l'architexte d'une communauté verbale (configuration des modèles de genres textuels disponibles dans cette communauté).

Ces genres sont porteurs d'indexations sociales. Ils ont la réputation d'être adaptés à (et pertinents pour) tel type d'activité (indexation référentielle), et d'être compatibles avec tel moyen d'interaction et de diffusion (indexation communicationnelle) ; par ailleurs, ces genres et leur maîtrise sont plus ou moins valorisés dans un groupe (indexation culturelle).

4. 2. Un modèle de l'architecture textuelle

Nous avons proposé des versions successives d'un modèle qui constitue en fait une esquisse théorique en permanence à retravailler (Bronckart et al, 1985 ; Bronckart, 1997 ; 2008). Fondé sur l'analyse quantitative des distributions d'unités linguistiques attestables dans un vaste corpus de textes du français contemporain (plusieurs milliers d'items), ce modèle vise à conceptualiser les opérations dont ces unités constituent les traces, et il est organisé en trois niveaux.

Le premier niveau concerne l'infrastructure du texte (fortement dépendante du genre dont ce texte relève) et a deux composants. L'un est centré sur la dimension thématique et son 
organisation: il a trait aux univers sémantiques mobilisés et aux conditions de leur déploiement en séries isotopiques de sèmes, ainsi qu'aux formes de planification articulant l'ensemble des univers sémantiques convoqués. L'autre a trait aux types de discours qui se combinent selon des modalités diverses au sein d'un genre. Ces types de discours correspondent à ce que Genette (1986) qualifiait de "modes de discours ", à savoir des formes d'organisation énonciative marquées par des configurations d'unités relativement stables (impliquant surtout les marques d'agentivité et d'organisation temporelle). II s'agit donc de formes d'organisation qui sont infra-ordonnées eu égard aux genres et qui sont en nombre limité ; notre modèle en retient quatre, le discours interactif, le discours théorique, le récit interactif et la narration.

Le deuxième niveau concerne les opérations visant à assurer la cohérence thématique d'un texte en dépit de l'éventuelle hétérogénéité (thématique et/ou discursive) de son infrastructure ; il s'agit en l'occurrence des opérations de connexion (se décomposant en diverses sous-opérations et marquées par des séries isotopiques d'organisateurs textuels) et des opérations de cohésion nominale (ayant trait aux modalités de gestion de la co-référence et marquées par les procédés de reprise anaphorique). Ce niveau présente un caractère "intermédiaire" dans la mesure où, si les opérations concernées sont par principe susceptibles de s'appliquer au texte dans son entier, les unités de marquage varient quant à elles selon le type de discours au sein duquel elles apparaissent.

\begin{tabular}{|c|c|c|c|}
\hline \multicolumn{2}{|c|}{ INFRASTRUCTURE } & \multirow{2}{*}{$\begin{array}{l}\text { COHERENCE } \\
\text { THEMATIQUE }\end{array}$} & \multirow{2}{*}{$\begin{array}{l}\text { COHERENCE } \\
\text { INTERACTIVE }\end{array}$} \\
\hline \multirow{9}{*}{$\begin{array}{l}\mathrm{P} \\
\mathrm{L} \\
\mathrm{A} \\
\mathrm{N} \\
\mathrm{I} \\
\mathrm{F} \\
\mathrm{I} \\
\mathrm{CA} \\
\mathrm{T} \\
\mathrm{I} \\
\mathrm{O} \\
\mathrm{N}\end{array}$} & & & \\
\hline & & Processus isotopiques & Engagement énonciatif \\
\hline & TYPES DE & & \\
\hline & DISCOURS & & FOYER \\
\hline & EMBOÎTES & CONNEXION & ENONCIATIF \\
\hline & & & \\
\hline & EVENTUELLES & & \\
\hline & SEQUENCES & & GESTION DES VOIX \\
\hline & & COHESION & \\
\hline
\end{tabular}

Figure 1. Schéma de l'architecture textuelle 
Le troisième niveau concerne les opérations assurant la cohérence interactive d'un texte, c'est-à-dire visant à expliciter les instances assumant la responsabilité du contenu thématique, ainsi que les évaluations portées sur l'un ou l'autre élément de ce même contenu. Sont ici concernés: - les modalités de confection du foyer énonciatif, comme instance formelle gérant l'ensemble des engagements interactifs dont témoigne le texte ; les divers types de voix qui sont mises en scène et distribuées dans le texte ; - les diverses modalisations (épistémique, déontique, appréciative, etc.) explicitant les évaluations ou les jugements portés sur certains aspects du contenu.

\section{3. Statut et rôle des types de discours}

Tels que nous les définissons (cf. Bronckart, 1997, p. 137-218), les types de discours sont des configurations d'unités et de processus linguistiques relativement stables, infraordonnées eu égard aux genres textuels et traduisant une "attitude discursive globale" que nous qualifions de monde discursif. Ces mondes discursifs se construisent sur la base de deux types d'opérations. Les premières explicitent le rapport existant entre les coordonnées organisant le contenu thématique d'un texte et les coordonnées du monde externe dans lequel se déploie l'action langagière dont le texte est issu. Les secondes ont trait à la mise en rapport entre, d'une part les différentes instances d'agentivité (personnages, groupes, institutions, etc.) et leur inscription spatio-temporelle, telles qu'elles sont mobilisées dans un texte, et d'autre part les paramètres matériels de l'action langagière en cours (agent producteur, interlocuteur éventuel et espace-temps de production).

Pour le premier type d'opération, soit les coordonnées du monde discursif sont présentées comme clairement disjointes de celles du monde de l'action langagière, soit cette mise à distance n'est pas opérée, et les deux sortes de coordonnées sont dès lors nécessairement conjointes. Dans le premier cas, que les thèmes mobilisés concernent des faits passés et attestés, des faits à venir, plausibles ou purement imaginaires, leur organisation s'ancre dans une origine temporelle qui spécifie le type de disjonction opérée (un jour, hier, en l'an 2058, etc.). Les faits organisés à partir de cet ancrage sont alors racontés "comme s'ils étaient passés". Dans le second cas, les thèmes mobilisés, dès lors qu'ils ne s'ancrent dans aucune origine temporelle, s'organisent inévitablement en référence plus ou moins directe aux coordonnées du monde de l'action langagière. Les faits sont alors présentés comme demeurant accessibles dans ce monde ; ils ne sont pas racontés, mais sont montrés, ou encore exposés. Cette première distinction revient ainsi à distinguer les mondes de l'ordre $d u$ RACONTER et les mondes de l'ordre de l'EXPOSER.

Pour le second type d'opération, soit un segment de texte explicite le rapport que ses instances d'agentivité entretiennent avec les paramètres matériels de l'action langagière 
(agent producteur, interlocuteur éventuel, et leur situation dans l'espace-temps), soit ce rapport n'est pas explicité et les instances d'agentivité de ce segment entretiennent alors un rapport d'indifférence avec les paramètres de l'action langagière en cours. Dans le premier cas, le texte mobilise ou implique les paramètres de l'action langagière, sous la forme de renvois déictiques à ces mêmes paramètres. Dans le second cas, le texte se présente dans une relation d'autonomie à l'égard des paramètres de l'action langagière. Une seconde distinction générale peut ainsi être posée entre les mondes discursifs exhibant, soit un rapport d'implication, soit un rapport d'autonomie, à l'égard des paramètres de l'action langagière.

En croisant ces deux distinctions, on aboutit à l'identification de quatre mondes discursifs, qui sont traduits par ces configurations d'unités et de processus linguistiques que nous qualifions de types de discours : le monde de l'EXPOSER impliqué se réalise en discours interactif, le monde de I'EXPOSER autonome en discours théorique, le monde du RACONTER impliqué en récit interactif et le monde du RACONTER autonome en narration.

Coordonnées générales des mondes

Conjonction Disjonction

EXPOSER RACONTER

\section{Rapport Implication Discours interactif Récit interactif \\ à l'acte de \\ production Autonomie Discours théorique Narration}

Figure 2. L'organisation des quatre types de discours

Selon notre approche, les types de discours servent de lieu d'interface entre les représentations individuelles (ayant leur siège en un organisme-agent) et les représentations collectives (ayant leur siège dans les œuvres humaines), et ils constituent les formats obligés de cette mise en interface.

Lorsqu'il (re-)produit un type de discours, un individu doit procéder à la planification interne des segments concernés, et il apprend ainsi à mettre en œuvre ces processus indissolublement mentaux et langagiers que sont les raisonnements : raisonnements pratiques impliqués dans les interactions dialogales ; raisonnements causaux-chronologiques impliqués dans les récits et les 
narrations (cf. Ricœur, 1983) ; raisonnements d'ordre logique et/ou semi-logique impliqués dans les discours théoriques (cf. Grize, 1984).

Cette médiation par les types constitue un processus de développement fondamental, dans la mesure où c'est par elle que se transmettent les grandes formes d'opérativité de la pensée humaine.

\section{Le rôle du langage dans la réflexivité}

Dans ce qui suit, nous commenterons quelques résultats obtenus dans le cadre de recherches que nous conduisons dans le domaine de la formation des adultes, qui visent à analyser les fonctions et le rôle que jouent les opérations langagières, dans la gestion des tâches de travail d'une part, dans l'élaboration et le développement des représentations de cette tâche (et plus généralement du travail et/ou du métier) d'autre part. Les données que nous utiliserons sont issues d'entretiens réalisés dans un hôpital et dans une institution de formation d'enseignants.

L'analyse de ces entretiens a exploité le modèle de l'architecture textuelle (cf. 4.2, supra) et s'est déployée en allant du texte vers les unités de rang inférieur : types de discours $\rightarrow$ relations prédicatives et mécanismes de cohésion $\rightarrow$ unités minimales de l'ordre du mot. Dans le cadre de cette analyse, nous avons accordé une attention toute particulière à ces modalités d'organisation énonciative que sont les types de discours, en examinant leur rôle dans le processus interprétatif de l'agir suscité par les entretiens. L'examen de ce qui se passe lorsque ces types de discours organisent un contenu thématique de l'ordre de l'agir a permis de mettre en évidence cinq configurations interprétatives, que Bulea a qualifiées de figures d'action (cf. Bulea, 2007 ; 2009 ; Bulea \& Fristalon, 2004)

\section{1. L'action occurrence}

\section{Exemple : travail infirmier}

«non dans ma tête j'essayais deee / j'ai j'ai j'ai re-visualisé / la façon dont le médecin avait fait [EB : han han] juste la partie heu où il utilisait les pinces et pis après j'ai essayé deee // de penser comment est-ce que moi j'allais pouvoir heu [IF : mum mum] [EB : mum mum] comment est-ce que j'allais pouvoir faire / tout en me disant dans la chambre peut-être que j'y arriverais pas avec les pinces mais que c'était pas // pas grave // (sourires) puis que j'allais prendre mon temps »

La figure de l'action occurrence constitue une saisie de l'agir en tant que contigu à sa mise en forme langagière et se caractérise ainsi par un très fort degré de contextualisation. Sa construction repose sur l'identification d'un ensemble d'ingrédients de l'agir saisis dans leurs dimensions particulières, spécifiques. 
Cette figure apparaît quasi exclusivement dans des segments de discours interactif, avec un axe de référence temporelle qui est celui de cette même situation. Du point de vue des marques d'agentivité, l'infirmière est régulièrement désignée par des je, ce qui signale sa forte implication, ou son statut d'acteur. Enfin, cette figure se caractérise par un nombre important de relations prédicatives indirectes, dont $70 \%$ environ constituent des modalisations pragmatiques. Elle comporte également de nombreuses modalisations externes aux relations prédicatives, dont la plupart sont des modalisations déontiques («il faut que je regarde »).

\subsection{L'action événement passé}

\section{Exemple : enseignement (Instruction au sosie)}

«S : à quel moment je prends ce temps pour parler avec eux -

$P$ : à n'importe quel moment / je peux arrêter toute activité // par exemple hier il y en avait qui se tenaient comme ça / et j'ai pris un quart d'heure pour leur dire que je n'acceptais pas / que moi jamais une fois ils m'ont vu comme ça au bureau ou allongé sur une table / jamais / donc c'est du respect / que le respect c'est mutuel ou alors c'est rien / ça je peux arrêter tout »

La figure de l'action événement passé procède par délimitation et extraction (du passé) d'une unité praxéologique identifiée, saillante et illustrative de l'agir.

Cette figure apparaît dans des segments de récit interactif, les procès évoqués étant saisis en référence à un axe temporel situé en amont de la situation d'entretien, et dont l'origine est marquée (par exemple, hier). L'actant demeure impliqué dans l'événement raconté, cette implication étant marquée, comme dans la figure précédente, par la présence massive du pronom je. Cette figure se caractérise enfin par une structuration des faits racontés relevant du schéma narratif prototypique (situation initiale, complication, résolution, évaluation) qui confère à l'unité extraite sont statut d'« événement ».

\section{3. L'action expérience}

\section{Exemple : travail infirmier}

" j'fonctionne toujours comme ça / je vois si le patient est disponible dans la matinée s'y a pas d'examen s'y a pas de chose comme ça / j'préviens le patient qu'on va faire son pansement // des fois ça peut être dix minutes avant / des fois ça peut être une demie heure un heure // si c'est quelqu'un très angoissé on évite de le prévenir trop avant parce que sinon (rires) / mais / je préviens toujours avant le soin / après j'arrive ben si il est s'il est assis au fauteuil ben je le recouche je l'installe // je préserve la / la pudeur / puis j'installe mon matériel puis je fais le pansement » 
La figure de l'action expérience constitue une saisie de l'agir sous l'angle de la cristallisation personnelle de multiples occurrences d'agir vécus : elle propose une sorte de bilan de l'état actuel de l'expérience de l'actant eu égard à la tâche concernée.

Cette figure apparaît principalement dans des segments de discours interactif, mais avec un axe de référence temporelle non borné, marqué par des adverbes à valeur généralisante et réitérative (normalement, souvent, de toute façon, etc.). Son organisation discursive procède par juxtaposition de procès, qui tendent à reproduire l'ordre chronologique du soin, mais signalent aussi des points de bifurcation de l'agir. Du point de vue agentif, on observe le co-fonctionnement de plusieurs formes pronominales (je, $t u$, on), qui attestent d'une moindre implication de l'agent.

\section{4. L'action canonique}

\section{Exemple : travail infirmier}

« l'installation heu on déballe la plaie on prépare le / le set [IF : hum hum] / après on fait le pansement on communique / on ferme le pansement on réinstalle le patient on met la ceinture on réhabille / on remet bien le lit en position on lui propose d'aller au fauteuil si il doit aller au fauteuil ou il reste comme ça on lui remet tout son matériel à disposition / on tire le rideau et après / on on dit heu / voilà / au revoir au patient quoi »

La figure de l'action canonique réside en une saisie de l'agir sous forme de construction théorique, et elle propose une logique de la tâche présentée comme a-contextualisée, à validité générale. De ce fait, elle rend compte surtout de la structure chrono-logique prototypique de l'agir, ainsi que des normes qui le régissent.

Cette figure s'organise sous forme de discours théorique, ou de mixte théoriqueinteractif. L'axe de référence temporelle est non borné et généralement non marqué, et les procès sont saisis par des formes de présent générique, dont l'ordre tend à reproduire la chronologie générale du soin. Cet ordre chronologique est en outre exprimé au travers d'une organisation phrastique récurrente, correspondant à la structure canonique sujet verbe - complément.

\section{5. L'action définition}

\section{Exemple : travail infirmier}

« V : le soin des prises des constantes [INT : heu] ben ça dépend aussi des horaires c'est ce qu'on disait le matin à 8 h c'est vrai que c'est important parce que c'est là // c'est le premier contact de la journée en fait donc heu c'est une approche pour // comment s'est passée la nuit pour (...) parce que c'est important pour nous de // c'est aussi une demande médicale 
mais c'est un prétexte aussi pour entamer la journée auprès d'eux donc voilà ce qui y a de particulier »

La figure de l'action définition relève d'une saisie de l'agir en tant qu'objet de réflexion, ou "phénomène dans le monde". Contrairement aux autres figures, elle ne thématise ni les actants, ni l'organisation chronologique de l'agir, ni ses constituants praxéologiques, mais rassemble des traits jugés pertinents, susceptibles de le circonscrire et de le différencier d'autres sortes d'activité.

Cette figure est insérée dans des segments relevant du discours théorique ou du mixte théorique-interactif, mais contrairement à l'action canonique, les formes verbales mobilisées ne portent qu'exceptionnellement sur des actes ou des gestes. La grande majorité des relations prédicatives est constituée des constructions impersonnelles en c'est et il y a.

\section{Le rôle de la réflexivité dans l'apprentissage et le développement}

Deux remarques tout d'abord, l'une théorique, l'autre méthodologique.

Au plan théorique, il apparaît clairement que, dans les analyses de leurs pratiques, les travailleurs prennent certes parfois en compte des actions "situées" dans un espace-temps déterminé (figures de l'action occurrence ou de l'action événement passé), mais ils se centrent au moins aussi fréquemment sur des schémas d'action théoriques ou "a-situés", construits soit sur la base de leur expérience de travail (figure de l'action expérience), soit sur celle des procédures édictées par leur organisation d'appartenance (figure de l'action canonique). Et ils saisissent parfois encore l'action sous l'angle de la signification qu'elle revêt, pour leur environnement de travail ou pour eux-mêmes (figure de l'action définition). On peut donc s'interroger sur les raisons de l'accent porté dans certains cadres théoriques sur le caractère "situé" de l'action : si cet adjectif désigne une propriété de l'action concrète, alors il s'agit d'un truisme; et s'il désigne l'angle sous lequel l'action est nécessairement saisie dans les procédures de mise en intelligibilité, alors il s'agit d'une erreur.

$\mathrm{Au}$ plan méthodologique, il faut relever que certaines démarches d'analyse des pratiques peuvent échouer, notamment lorsque la lecture des actions est surdéterminée par des attentes ou des injonctions sur ce qui devrait être vu. Nous en avons une exemple flagrant dans une autre recherche, conduite au Brésil avec des futurs enseignants de langue qui, plutôt que d'analyser les actions de professeurs, les évaluent d'emblée en regard de principes énoncés dans des ouvrages de référence ou dans des cours 
théoriques. L'analyse des pratiques ne s'improvise donc pas et doit au contraire être soigneusement préparée.

\section{1. Les produits de la réflexivité}

Les figures d'action que nous venons de décrire sont en principe identifiables dans l'ensemble des textes d'analyse des pratiques, mais on ne peut exclure que des recherches portant sur d'autres milieux de travail, ou d'autres types de tâches, en fassent apparaître d'autres encore.

Ces figures constituent des variantes interprétatives de l'activité, qui en soi sont évidemment toutes pertinentes et légitimes, et qui se présentent dès lors comme des composantes du « conflit des interprétations » que thématisait Ricœur (1969).

Certains travailleurs se limitent à l'élaboration d'une ou de deux figures d'action, alors que d'autres se révèlent aptes à proposer, au cours d'un même entretien, l'ensemble de ces figures.

La confection de ces figures requiert un ensemble d'opérations langagières, dont le choix d'un type de discours, d'une forme de repérage temporel, d'une forme de codification des actants, etc. La maîtrise de ces opérations langagières apparaît ainsi comme une des conditions de possibilité de confection des différentes figures.

\section{2. De la réflexivité à l'apprentissage et au développement}

La réflexivité suscite la prise de conscience de certains aspects, ou de certaines dimensions, des pratiques sous analyse. Cette prise de conscience est une condition nécessaire pour une meilleure compréhension, ou pour un surcroît d'intelligibilité de l'agir, mais ce n'est néanmoins pas une condition suffisante: il peut arriver en effet qu'un surcroît de compréhension de "ce qui se passe" ne se traduise par aucun apprentissage, voir même engendre une manière de blocage ou de régression.

Nous émettrons l'hypothèse que la prise de conscience ne constitue un facteur de développement que dans la mesure où elle engendre une réorganisation positive et cohérente des significations de l'action; positive dans la mesure où elle met en perspective et tente d'intégrer les diverses variantes du débat interprétatif ; cohérente, dans la mesure où, sur fond de ce débat, la personne se construit une conception personnelle de son action.

Cette réorganisation des significations constitue un indice synchronique de ce que la personne s'est (re-)mise dans une dynamique interprétative ; dynamique dont l'issue n'est jamais assurée, et dont les effets sur l'efficacité et la pertinence des actions concrètes, n'est non plus jamais assurée, et est du ressort de la seule personne en développement. 


\section{Bibliographie}

Anscombe, G.E. (2001). Intention. Paris : Gallimard [Edition originale : 1957].

Bronckart, J.-P. (1997). Activité langagière, textes et discours. Pour un interactionisme socio-discursif. Paris : Delachaux et Niestlé.

Bronckart, J.-P. (2008). Genres de textes, types de discours et "degrés" de langue. Hommage à François Rastier. Texto, disponible sur http://www.revue-texto.net/.

Bronckart, J.-P. et al. (1985). Le fonctionnement des discours. Paris : Delachaux et Niestlé.

Bühler, K. (1927). Die Krise der Psychologie. Jena : Fischer.

Bulea, E. (2007). Le rôle de l'activité langagière dans l'analyse des pratiques à visée formative. Thèse de doctorat. Université de Genève.

Bulea, E. (2009). Types de discours et interprétation de l'agir: le potentiel développemental des "figures d'action". Estudos linguisticos ; Linguistic Studies (sous-presses).

Bulea, E. \& Frislaton, I. (2004). Agir, agentivité et temporalité dans des entretiens sur le travail infirmier. In : J.-P. Bronckart \& Groupe LAF (Ed.), Agir et discours en situation de travail. Cahiers de la Section des Sciences de l'Education (Genève), 103, 11-144.

Clot, Y. (2001). Clinique du travail et action sur soi. In J.-M. Baudouin \& J. Friedrich (Ed.), Théories de l'action et éducation (pp. 255-276). Bruxelles : De Boeck.

Dewey, J. (1925). Experience and Nature. New York : Dover.

Durkheim, E. (1898). Représentations individuelles et représentations collectives. Revue de métaphysique et de morale, 6, 273-302.

Genette, G. (1986). Introduction à l'architexte. In G. Genette et al., Théorie des genres (pp. 89-159). Paris : Seuil [Edition originale : 1979].

Giddens, A. (1987). La constitution de la société. Paris : PUF [Edition originale : 1984].

Grize, J.-B. (1984). Sémiologie du raisonnement. Berne : Peter Lang.

Habermas, J. (1987). Théorie de l'agir communicationnel, tome 1 : Rationalité de l'agir et rationalisation de la société. Paris : Fayard.

Leontiev, A.N. (1979). The Problem of Activity in Psychology. In J.V. Wertsch (Ed.), The Concept of Activity in Soviet Psychology (pp. 37-71). New-York : Sharpe.

Mead, G.H. (1934). Mind, self and society from the standpoint of a social behaviorist. Chicago : University of Chicago Press. 
Oddone, I., Re, A. \& Briante, G. (1981). Redécouvrir l'expérience ouvrière. Vers une autre psychologie du travail ? Paris : Editions sociales.

Piaget, J. (1970). Epistémologie des sciences de l'homme. Paris : Gallimard.

Ricœur, P. (1969). Le conflit des interprétations. Paris : Seuil.

Ricœur, P. (1977). Le discours de l'action. In P. Ricœur (Ed.), La sémantique de l'action. Paris : cnrs.

Ricœur, P. (1983). Temps et récit, t.1. Paris : Seuil.

Saussure, F. de (2002). Ecrits de linguistique générale. Paris : Gallimard.

Schön, D. A. (1983). Le praticien réflexif. A la recherche du savoir caché dans l'agir professionnel. Québec : Editions Logiques.

Schütz, A. (1998). Choisir parmi des projets d'action. In: Eléments de sociologie phénoménologique. Paris : L'Harmattan.

Vermersch, P. (1994). L'entretien d'explicitation en formation initiale et en formation continue. Paris : ESF.

Voloshinov, V.N. (1977). Marxisme et philosophie du langage. Paris : Minuit [Edition originale : 1929].

Vygotski, L.S. (1997). Pensée et langage. Paris : La Dispute [Edition originale : 1934]. 